\title{
The role of interface ions in the control of water permeation through a carbon nanotube
}

\author{
Yunzhen Zhao, ${ }^{1}$ Jingyi Chen, ${ }^{2}$ Decai Huang, ${ }^{1}$ Jiaye $\mathrm{Su}^{1 *}$
}

${ }^{1}$ Department of Applied Physics, Nanjing University of Science and Technology, Nanjing, Jiangsu 210094, China.

${ }^{2}$ School of Material Science and Engineering, Zhejiang University of Technology, Hangzhou, Zhejiang 310014, China.

*Electronic mail: jysu@iccas.ac.cn; jysu@njust.edu.cn;

\section{Supporting Information}

Table S1: Non-bonded parameters for all atom types in the main text.

\begin{tabular}{ccccc}
\hline Atom type & $\sigma_{i}(\AA)$ & $\epsilon_{i}(\mathrm{~kJ} / \mathrm{mol})$ & charge $(e)$ & ref \\
\hline $\mathrm{Na}^{+}$ & 3.2500 & 0.7113 & +1 & $\mathrm{~S} 1$ \\
$\mathrm{Cl}^{-}$ & 4.4010 & 0.4184 & -1 & $\mathrm{~S} 1$ \\
$\mathrm{C}$ & 3.3997 & 0.3598 & 0 & $\mathrm{~S} 1$ \\
$\mathrm{O}\left(\mathrm{H}_{2} \mathrm{O}\right)$ & 3.1506 & 0.6364 & -0.834 & $\mathrm{~S} 2$ \\
$\mathrm{H}\left(\mathrm{H}_{2} \mathrm{O}\right)$ & 0 & 0 & 0.417 & $\mathrm{~S} 2$ \\
$\mathrm{C}\left(\mathrm{COO}^{-}\right)$ & 3.5812 & 0.2774 & 0.27 & $\mathrm{~S} 3$ \\
$\mathrm{O}\left(\mathrm{COO}^{-}\right)$ & 2.6259 & 1.7249 & -0.635 & $\mathrm{~S} 3$ \\
$\mathrm{~N}\left(\mathrm{NH}_{3}^{+}\right)$ & 3.1365 & 0.6398 & 0.129 & $\mathrm{~S} 3$ \\
$\mathrm{H}\left(\mathrm{NH}_{3}^{+}\right)$ & 0 & 0 & 0.248 & $\mathrm{~S} 3$
\end{tabular}

The Lorentz-Bertelot rule is used to determine the parameters between different atoms, i.e., $\sigma_{i j}=\left(\sigma_{i i}+\sigma_{j j}\right) / 2$ and $\epsilon_{i j}=\left(\epsilon_{i i} \epsilon_{j j}\right)^{1 / 2}$. The charge of graphene carbon atom connecting to $\mathrm{NH}_{3}{ }^{+}$is $0.127 \mathrm{e}$.

\section{Part A: The effect of applied electric fields}

We pursue the water dynamics inside the CNT. Figure S1 gives the water translocation time and occupancy number as a function of the electric field for the two 
charged surfaces. The water translocation time is averaged from all successful translocation events, i.e., the flow events. Generally, a smaller translocation time will correspond to a larger water flow. As we can see in Figure S1a for the $\mathrm{COO}^{-}$system, the water translocation time exhibits a linear decay with $E$, corresponding to the flow and flux increase in Figure 2a of the main text. Interestingly, this linear decay is similar to the direct water-ion coupling transport through a wider CNT in electric fields [S4], suggesting widely existed water dynamics induced by the ion drag. For the $\mathrm{NH}_{3}{ }^{+}$system in Figure $\mathrm{S} 1 \mathrm{~b}$, the water translocation time exhibits fluctuations at small electric fields, similar to the flow fluctuation in Figure $2 b$ of the main text. Overly, the translocation time for $\mathrm{COO}^{-}$system is smaller than that for $\mathrm{NH}_{3}{ }^{+}$at the same electric field. This can also explain the higher water flow of $\mathrm{COO}^{-}$. From Figure S1 we can further see that the water occupancy $<\mathrm{N}>$ behaves almost linear reduction for both systems and can be ascribed to the ion drag. However, the occupancy variation is still very limited as the single-file water chain is maintained.

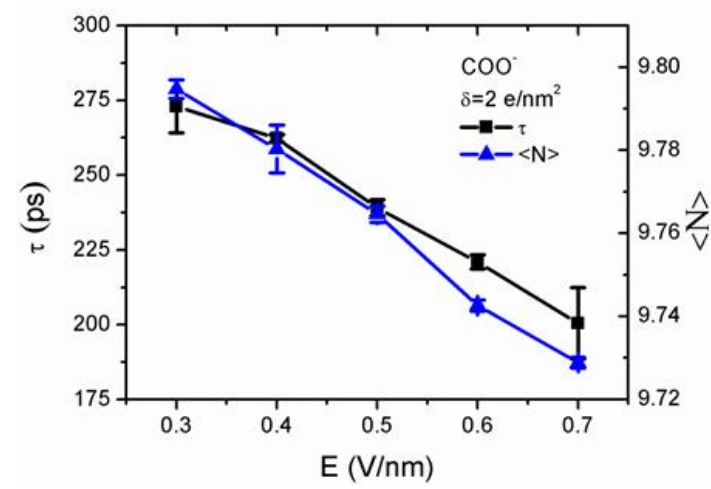

(a)

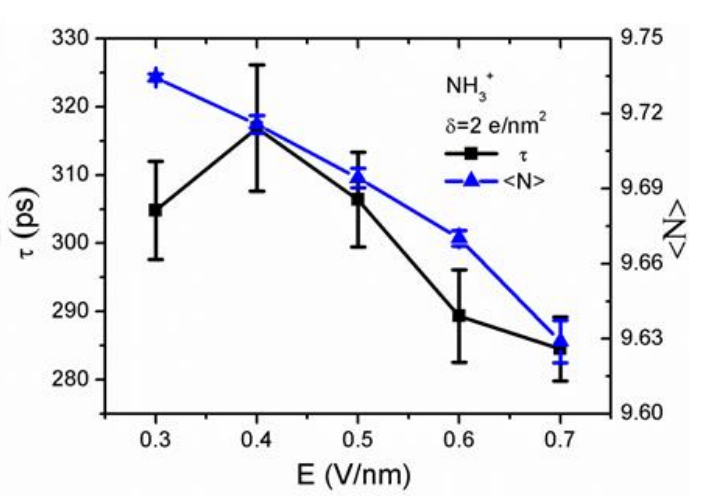

(a)

Figure S1. The water translocation time $\tau$ and occupancy $<\mathrm{N}>$ as a function of the electric field for the (a) $\mathrm{COO}^{-}$and (b) $\mathrm{NH}_{3}{ }^{+}$systems, respectively.

In order to understand the water distributions, we show in Figure S2 the axial density profiles and hydrogen bond (H-bond) number as a function of the water position along the CNT axes. Similar to previous work [S5-S7], we use $0.16 \mathrm{~nm}$ in the CNT center region as the effective diameter for the density computation, by taking into accounts the carbon-water Lennard-Jones potential. The distributions are almost independent of the field strength. The wave style patterns of water density inside the 
CNT are a sign of single-file water chain, stemming from the steric distribution of CNT atoms. The density inside the CNT is about four times of the bulk water, denoting the possible usage in mass storage. The ten density peaks are in accordance with the water occupancy in Figure S1. In addition, we also note that the density waves for $\mathrm{NH}_{3}{ }^{+}$are relatively distorted, and should be relevant to the extraordinary water dipole orientations (discussed in the main text). When a water molecule moves from bulk to the present CNT, it will lose two H-bonds on average, suggested by the $\mathrm{H}$-bond distributions in Figure S2. This energy penalty will be partially compensated from the water-CNT hydrophobic interaction. Besides, compared to the stable $\mathrm{H}$-bond networks in bulk, the water molecules inside the CNT will have more freedom to rotate, and thus gain some rotational entropy [S8,S9], interpreting the CNT filling. Thus, the water molecules are quite different from some macromolecules, such as proteins, DNAs or polymers, whose entropy should be reduced in confined environment. Similarly, the H-bond distributions are also slightly distorted for the $\mathrm{NH}_{3}{ }^{+}$system.



(a)

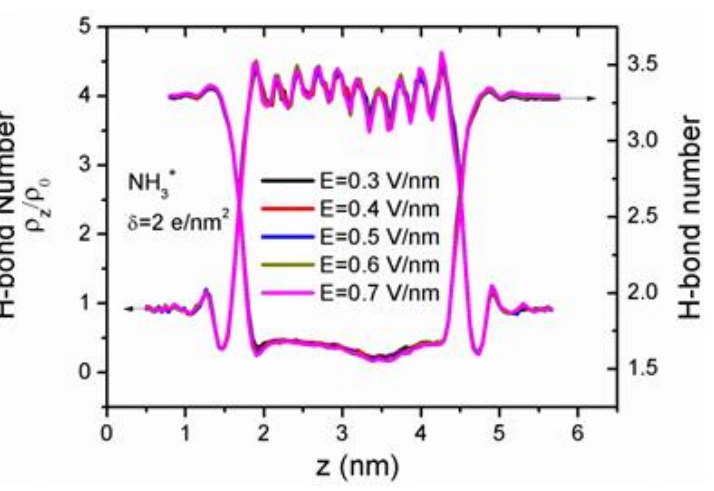

(b)

Figure S2. The axial water density profiles and hydrogen bond number as a function of $\mathrm{z}$ position along the CNT for the (a) $\mathrm{COO}^{-}$and (b) $\mathrm{NH}_{3}{ }^{+}$systems. $\rho_{0}=1.0 \mathrm{~g} / \mathrm{cm}^{3}$ is the bulk water density.

\section{Part B: The effect of charged residue density}

We further present in Figure S3 the water translocation time and occupancy as a 
function of the charge density. Clearly, the translocation time for the $\mathrm{COO}^{-}$and $\mathrm{NH}_{3}{ }^{+}$ systems both increase to plateaus, corresponding to the flow behaviors in Figure 5 of the main text. Accordingly, the $\mathrm{COO}^{-}$system also has smaller translocation time, matching its larger flow values. The water occupancy $<\mathrm{N}>$ of $\mathrm{COO}^{-}$system shows a monotonous decay with the charge density, since the ion pulling should lead to a trend of CNT empty; while it has larger fluctuations at high charge density for the $\mathrm{NH}_{3}{ }^{+}$ system. Nonetheless, due to the preservation of single-file water chain, the variation of $<\mathrm{N}>$ is still very limited for both systems.

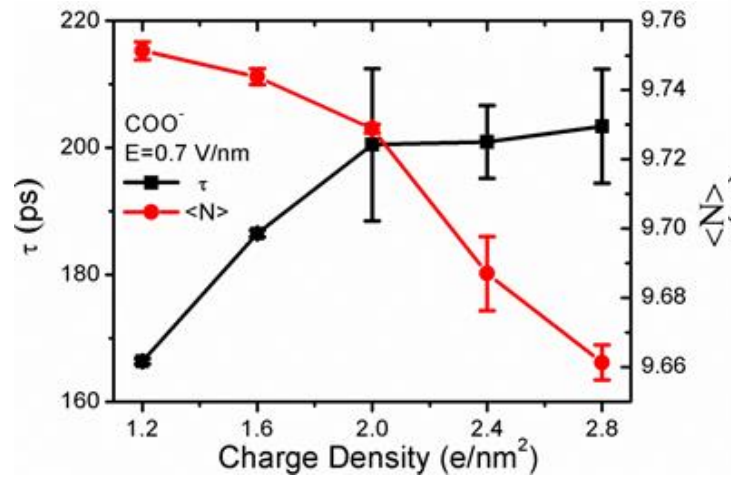

(a)

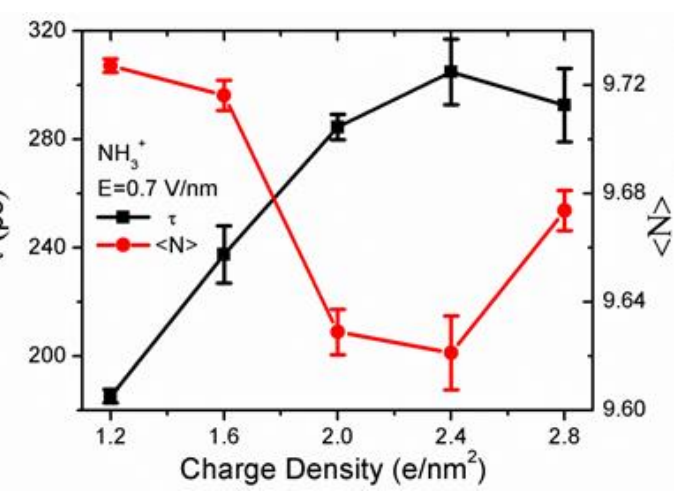

(b)

Figure S3. The water translocation time and occupancy $<\mathrm{N}>$ as a function of the charge density for the (a) $\mathrm{COO}^{-}$and (b) $\mathrm{NH}_{3}{ }^{+}$systems.

Figure S4 shows the axial water density profiles and H-bond number as a function of the water position along the CNT axes for the two systems. As we can see that for the $\mathrm{COO}^{-}$system, the charge density does not have appreciable impact on the density and H-bond distributions, resembling the effect of electric fields. However, for the $\mathrm{NH}_{3}{ }^{+}$system, with the increase of charge density, the density and H-bond distributions change from normal to distorted patterns. For $\delta=1.2$ and $1.6 \mathrm{e} / \mathrm{nm}^{2}$, the wave-like density profiles and H-bond distributions are normal; however, the distributions become distorted and even swollen at high charge density. Clearly, according to the above analyses the unique water dipole orientation should be the major reason for uncovering these abnormal distributions. Overly, the surface charge density has more profound impact than electric fields on the thermal dynamics of the single-file water chains. 


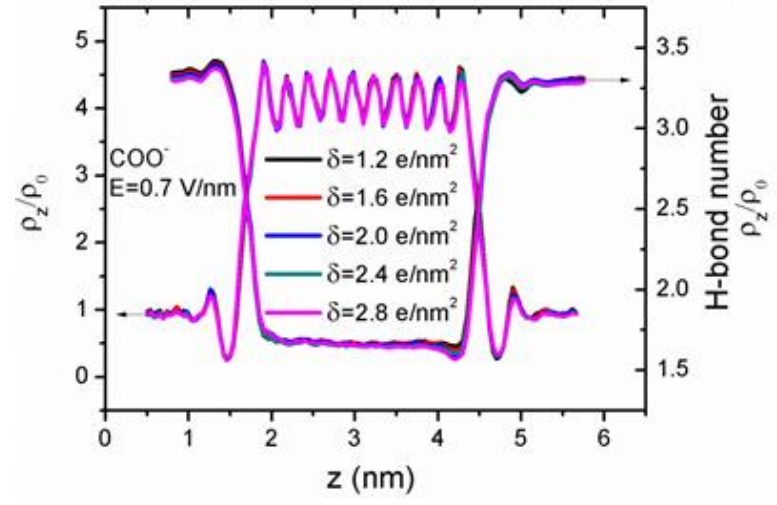

(a)

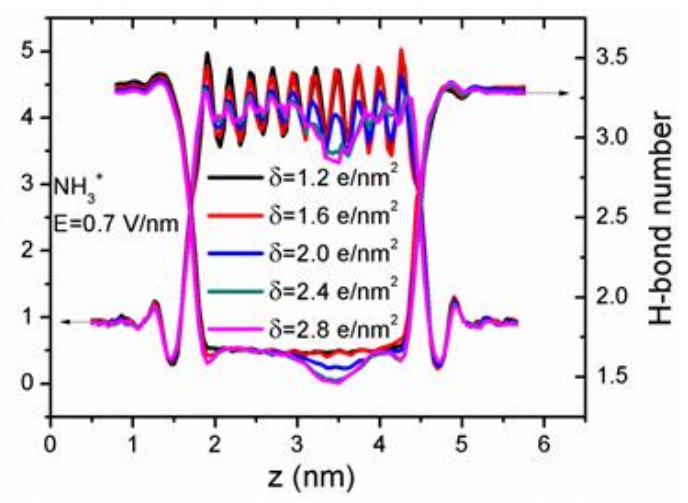

(b)

Figure S4. The axial water density profiles and hydrogen bond (H-bond) number as a function of $\mathrm{z}$ position along the $\mathrm{CNT}$ for the (a) $\mathrm{COO}^{-}$and (b) $\mathrm{NH}_{3}{ }^{+}$systems. $\rho_{0}=1.0$ $\mathrm{g} / \mathrm{cm}^{3}$ is the bulk water density.

\section{REFERENCES}

[S1] Duan, Y.; Wu, C.; Chowdhury, S.; Lee, M. C.; Xiong, G.; Zhang, W.; Yong, R.; Cieplak, P.; Luo, R.; Lee, T. A point-charge force field for molecular mechanics simulations of proteins based on condensed-phase quantum mechanical calculations. $J$. Comput. Chem. 2003, 24, 1999-2012.

[S2] Jorgensen, W. L.; Chandrasekhar, J.; Madura, J. D.; Impey, R. W.; Klein, M. L. Comparison of simple potential functions for simulating liquid water. J. Chem. Phys. 1983, 79, 926-935.

[S3] Schmid, N.; Eichenberger, A. P.; Choutko, A.; Riniker, S.; Winger, M.; Mark, A. E.; van Gunsteren, W. F. Definition and testing of the gromacs force-field versions 54a7 and 54b7. Eur. Biophys. J. 2011, 40, 843-856.

[S4] Su, J. Y.; Huang, D. C. Coupling transport of water and ions through a carbon nanotube: the role of ionic condition. J. Phys. Chem. C 2016, 120, 11245-11252.

[S5] Su, J. Y.; Guo, H. X. Control of unidirectional transport of single-file water molecules through carbon nanotubes in an electric field. ACS Nano 2011, 5, 351-359. [S6] Su, J. Y.; Yang, K. D. Temperature dependence of the transport of single-file water molecules through a hydrophobic channel. J. Comput. Chem. 2016, 37, 
$1043-1047$.

[S7] Su, J. Y.; Zhao, Y. Z; Fang, C.; Bilal Ahmed, S.; Shi, Y. Interface Nanoparticle Control of a Nanometer Water Pump. Phys. Chem. Chem. Phys. 2017, 19, 22406-22416.

[S8] Pascal, T. A.; Goddard, W. A.; Jung, Y. Entropy and the driving force for the filling of carbon nanotubes with water. Proc. Natl. Acad. Sci. U. S. A. 2011, 108, 11794-11798.

[S9] Kumar, H.; Mukherjee, B.; Lin, S. T.; Dasgupta, C.; Sood, A. K.; Maiti, P. K. Thermodynamics of water entry in hydrophobic channels of carbon nanotubes. $J$. Chem. Phys. 2011, 134, 124105. 